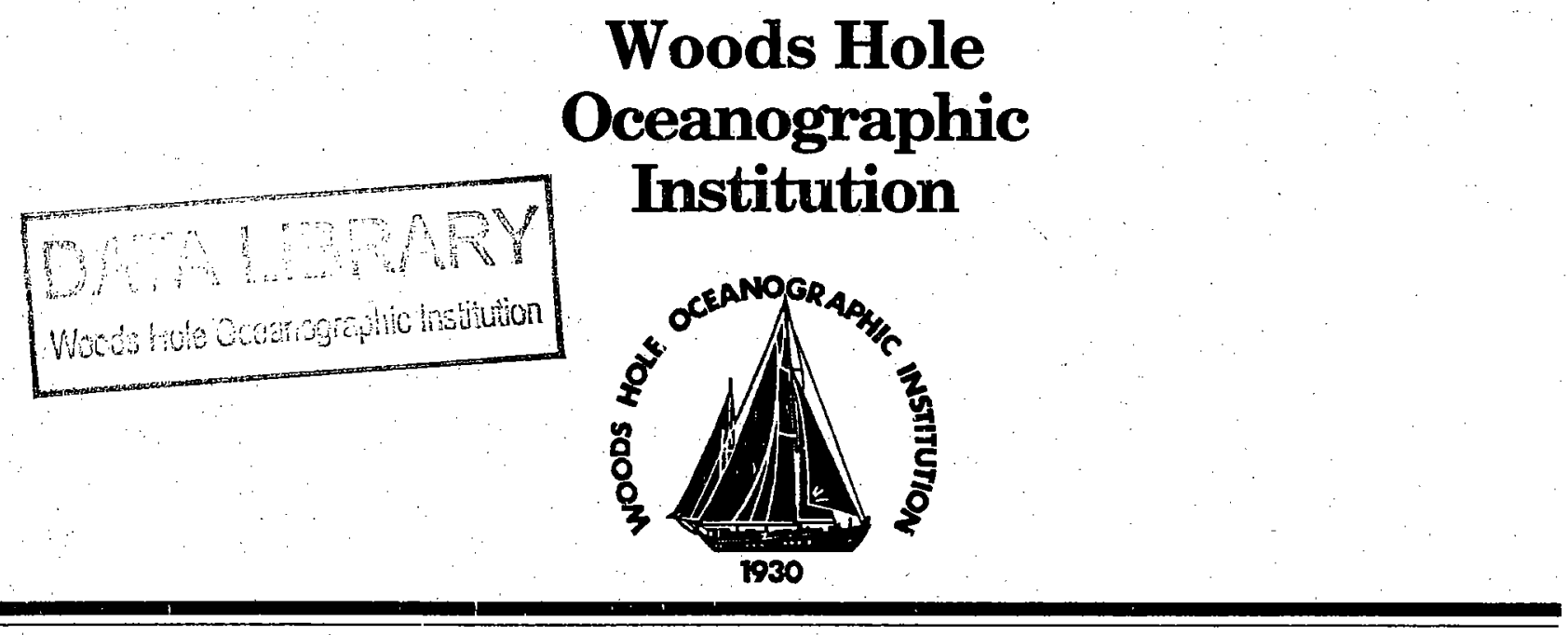

\title{
Marine Animal Sound Classification
}

\author{
by \\ Kurt M. Fristrup and William A. Watkins
}

October 1993

Technical Report

Funding was provided by the Office of Naval Research through the Naval Undersea Warfare Center under Contract No. N-00140-90-D-1979.

Approved for public release; distribution unlimited. 
WHOI-94-13

\title{
Marine Animal Sound Classification
}

\author{
by
}

Kurt M. Fristrup and William A. Watkins

Woods Hole Oceanographic Institution

Woods Hole, Massachusetts 02543

October 1993

\section{Technical Report}

Funding was provided by the Office of Naval Research through the Naval Undersea Warfare Center under Contract No. N-00140-90-D-1979.

\begin{abstract}
Reproduction in whole or in part is permitted for any purpose of the United States Government. This report should be cited as Woods Hole Oceanog. Inst. Tech. Rept., WHOI-94-13.
\end{abstract}

Approved for public release; distribution unlimited.

\section{Approved for Distribution:}

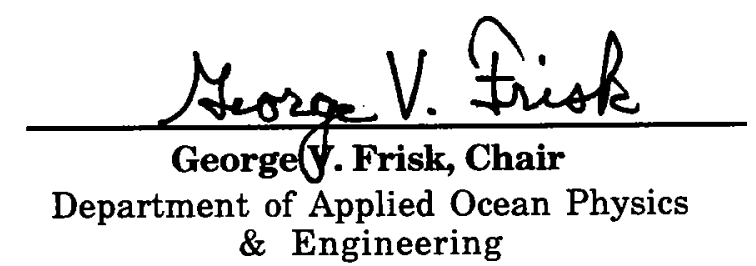




\section{Table of Contents}

$\begin{array}{ll}\text { Summary } & 1\end{array}$

Table of Contents 3

$\begin{array}{ll}\text { Introduction } & 4\end{array}$

$\begin{array}{lc}\text { The Feature Extraction Algorithm } & 6\end{array}$

$\begin{array}{lr}\text { Classification Performance } & 10\end{array}$

$\begin{array}{ll}\text { Literature Cited } & 13\end{array}$ 


\section{Introduction}

Marine animals produce a remarkable variety of sounds (Watkins and Wartzok 1985). A primary goal of our bioacoustic program at the Woods Hole Oceanographic Institution (WHOI) has been to parse this variation into biologically significant classes of signals. Marine mammal sounds exhibit distinctive features associated with species (op. cit.), individual identity (Caldwell, Caldwell and Tyack 1990), and certain behaviors. These features have never been examined quantitatively, comparing the sounds of a wide variety of species. Do these sounds remain distinctive as the scope of comparison broadens? Experienced researchers can aurally and visually (via spectrographic analysis) identify acoustic features that appear to be species-specific, and sometimes features unique to individual animals; can we specify numerical algorithms that objectively recognize these distinctions?

The logistic requirements for addressing these questions remain formidable. Many biological and environmental conditions potentially contribute to acoustic variability. To quantify the interspecific and intraspecific variability in marine animal sounds, large numbers of sounds must be accumulated and analyzed for each distinct class of sounds. Several results indicate that correct identification of sounds is significantly improved by utilizing all available biological information during the construction or "training" of the classifier (Fristrup and Watkins 1992). Therefore, numeric features extracted from acoustic data must be conveniently referenced to species, population, group, social context, behavior, activity, individual identity, sex, reproductive situation, age, season, geographic location, water depth, and sound propagation.

The SOUND database system of marine animal sounds (Watkins, Fristrup, and Daher 1991) provided this capability. The databases and associated files contained thousands of digitized sound segments. The database described the time, geographic location, recording conditions, identity of the animal(s) producing the sounds, the behavioral observations associated with sound production, etc. These SOUND databases represented years of work by several people. The ONR Ocean Acoustics Program (Marshall Orr) provided the initial funding, but continued development and expansion were funded by a blend of Navy and private sources. The feature extraction and classification program would not have been feasible without the SOUND resources. In turn, development of feature extraction and sound classification, funded by TRICCSMA, resulted in significant structural improvements in the database systems. New, relational database structures were implemented to permit flexible and convenient integration of statistical results with the biological and environmental information.

The ability to select and to analyze acoustic measurements based on related biological or environmental observations was crucial for these data. This could have been done by segregating data files for different species, activities, locations, etc. and independently processing each batch. However, it would have been increasingly cumbersome and difficult to manage data segregation as the scope and complexity of analyses increased. Maintaining the integrity of the data (correct file assortment by attributes, labeling processed output) would be problematic. A more powerful technique was to process all 
sound cuts in one batch, and to attach an identifier to the vector of measurements from each sound cut. Automatic feature extraction for all available sound cuts proceeded unattended, with one command. The resulting measurements, with the attached identifier, were imported as a table in a relational database program. The identifier provided a unique link between the vector of quantitative acoustic features describing a sound and the biological observations associated with that sound. Interactive exploration of relationships among statistics and biological or environmental factors followed, exploiting the convenience and flexibility of relational database queries.

The SOUND text databases for the recordings and the digital sound sequences (Watkins, Fristrup, and Daher 1991) could have accommodated new numeric data from the statistical analyses, but the INMAGIC software used to develop this system was unsuitable. It required restructuring the entire database each time the number of numeric fields changed. This was not feasible: the analyses required many iterations and modifications. Therefore, PARADOX software (relational database support, with visual, query-by-example interface) was used. The text information from the SOUND databases remained unmodified as distinct tables, and additional tables were created for the acoustic results. Statistical summaries of subsamples were generated with specific queries. This structuring of information also permits queries using sound characteristics to identify species and locations that have previously exhibited similar sounds.

The acoustic feature extraction program (AcouStat) was called with one command line parameter, the name of the file containing a digital sound cut. AcouStat processed these data, and sent the results to standard output (stdout in the $C$ language). Redirection of this output was used to store the data, or to pipe the acoustic features to another program for additional processing. For the analyses described here, these data were appended to a text file that was later imported into a PARADOX table. PARADOX queries were used to link text and acoustic features, and the results were exported to SPLUS and SYSTAT (data analysis packages) for classification analysis.

There is no scientific precedent for quantification of time-frequency characteristics of animal sounds on this scale. No prior work has dealt with so many species and such a variety of repertoires from individual animals. The WHOI studies of marine animal acoustics, a continuous program initiated by William E. Schevill in the late 1940's, have provided the heuristic basis for selecting features and designing algorithms. Our personnel utilize many different acoustic features to describe sounds and diagnose their identity. As a first step toward the development of an automatic, objective system for identifying animal sounds, we devised statistical measures to resolve familiar acoustic features. 


\section{The Feature Extraction Algorithm}

There is no a priori basis for selecting statistics that will maximize classifier performance.

Our approach has been iterative, guided by the following criteria:

- Each statistic was designed to emphasize particular parameters of animal sounds that we recognized as important for distinguishing species.

- Each statistic had to be insensitive to temporal artifacts introduced by ocean propagation (multipath, fading).

- Most statistics had to be relatively insensitive to noise levels (resistant to outliers, possessing a high breakdown point).

- Most statistics had to yield consistent results despite variation in the shape of the ambient noise power spectra.

- Many statistics needed to relate to obvious features in time-frequency displays of these sounds (duration, frequency range, ...). This eased interpretation of success and diagnosis of flawed performance.

The signal processing was relatively simple, using power spectra derived from a Fast Fourier Transform. For most files, FFT size was 256 sample points, but for very short files (low sampling rates) the FFT size was decreased to obtain a minimum of 16 FFT data blocks per file. Adjacent blocks overlapped by $25 \%$. The samples were level-shifted to obtain a block mean of zero, tapered with a Hamming window, and level shifted again to remove the DC bias introduced by tapering. The complex FFT values were multiplied by their complex conjugates (to form the magnitude-squared values), and the energy in the "negative" frequency bins was added to the corresponding "positive" frequency bins. Thus, the sum of the first $\mathbf{N}_{\mathrm{ft}} / 2+1$ bins equaled the sum-squared energy. Windowing smoothed the power spectra. Overlapping increased time resolution, and extracted useful information from data that would otherwise have been "lost" in the tails of the window.

More precise time-frequency analyses could be substituted for this procedure (Wigner-Villle, RID), but resolution of signal characteristics on these scales would be sensitive to phase perturbations. These techniques might require explicit source extraction (environmental deconvolution) to provide signals of sufficient quality. Such efforts were not indicated in the course of our analyses, but they remain an attractive option for very brief signals.

Noise Compensation

Our noise compensation technique starts with an estimated "average" noise power spectrum. This was computed as the median of the data blocks comprising the initial and terminal $5 \%$ of the sound file. By convention, our transient extraction protocols included a leader and trailer of background sound. Some signal energy was occasionally present in one of these regions, but the median spectrum was not grossly inflated by these signals. Previously, we used a large number of data blocks, taken at fixed intervals throughout the sound cut, and computed a noise spectrum from the quietest sections. The newer routine is comparable and faster. 
A multiple of this noise spectrum is subtracted from each data block's spectrum; all negative results are set to zero. Previously, we subtracted a constant multiple -- about $7 x$ the noise spectrum -- from all data blocks. This multiple was subjectively determined by examining a variety of spectrographs. The newer technique was "adaptive" because the level of the noise spectrum was adjusted prior to subtraction from each block's spectrum. This enhancement was prompted by frequent observations of "swelling" noise backgrounds: the shape of the noise spectrum seemed relatively constant, but the noise energy fluctuated widely in some cuts. Initial attempts to model this utilized orthogonal decompositions to identify principal components of noise spectrum variability. Utilization of more than two orthogonal components was found to introduce spectrographic artifacts in the form of frequency banding, due to the partial correlation of some noise vectors with transient sound spectra (biological signals). Also, the most significant improvement was seen to result from allowing the first component (essentially the mean spectrum) to vary. Thus, a simplified algorithm was devised.

Each bin in a data block's spectrum was divided by the corresponding bin in the noise spectrum, yielding a vector of possible multipliers. "Multipliers" indicates that if the noise spectrum is multiplied by one of these values, and subtracted from the data block's spectrum, the corresponding bin in the data spectrum will be exactly cancelled. These values were sorted, and the value corresponding to the 6th percentile order statistic (8th of 128) was used. This multiplier always underestimates the proper scaling for the noise spectrum, but it also is very unlikely to be inflated by signal energy: it is a consistent underestimate. This order statistic must be magnified by a constant value for best performance. The magnitude of that adjustment was determined by analyzing noise compensation performance with a variety of parameter settings.

To measure noise compensation performance, we needed to measure the relative amounts of noise and signal energy that were removed. All of our sound cuts represented single channel recordings containing noise and signal. Thus, synthetic signals resembling marine mammal sounds were generated, as were randomly generated noise sequences resembling the backgrounds in our cuts. The noise compensation algorithm was applied to pure signal and noise sequences respectively, and the residual energy after compensation was measured. Values were sought that preserved as much signal energy and removed as much noise energy as possible.

Before discussing the results, our index of performance merits explanation. Residual signal-to-noise ratio did not prove to be a useful measure, because this metric resulted in excessively high levels of noise subtraction. The "optimal" values reached with this metric resulted in spectrographs that retained only the very loudest portion of the signal. Critically important components of the signal (for classification) were subtracted out. A more useful metric proved to be the percent signal energy remaining minus the percent noise energy remaining. A simple interpretation provides heuristic justification for this criterion. One estimate of expected residual signal energy is the residual noise energy multiplied by the original signal-to-noise ratio. This would be accurate if both signal and noise energy were reduced in proportion by the noise compensation technique. Our 
metric is proportional to the "signal excess", the actual residual signal energy minus the expected residual signal energy. Optimal parameter values derived with this metric agreed with subjective judgments of spectrograph quality by experienced observers.

The most problematic sounds were relatively broad band, because more of the possible multipliers could be inflated by signal energy. This suggested that the best performance would be realized with low order statistics. Figure 1 presents the results of a simulation that used a broad-band signal and noise generated by forcing a sixth order autoregressive model with normally distributed white noise. Higher levels of signal excess represent better performance. Each vertical line represents performance at varying multiplier values, holding the order statistic constant. The leftmost vertical line starts at the bottom with a multiplier of 8 , and ends with a multiplier of 160 . The diagonal segment to the next vertical line denotes the performance value with the largest multiplier value on the left, and the performance of the next order statistic with its smallest multiplier on the right. Successive vertical lines represent different ranges of multipliers, ending with a range of $1->20$ for the $50 \%$ order statistic. The graph illustrates the falling levels of performance with increasing order statistic number, and our success in bracketing the best multiplier values for each order statistic. On the basis of these and other tests, we chose the 6th percentile order statistic and a multiplier value of 75 .

Figures 2 and 3 illustrate the effect of noise compensation, and compare the fixed noise compensation technique used previously with the adaptive technique. Both of these signals have poor signal-to-noise ratios, much worse than our typical sound cut. Note the improved retention of signal energy: fewer dropouts in the Lagenodelphis whistles, clearer representation of the soft, introductory moan in the right whale signal. The marked speckling in the Lagenodelphis adaptive spectrograph also represents preserved signal energy: echolocation clicks.

This noise compensation algorithm, and the methods we used to develop and test it, represented significant improvements over our previous work, but we do not represent this as the optimal or state-of-the-art technique. It allows us to achieve impressive classification performance. In our judgment, further improvements in this area are desirable, but not essential. The software has been designed to facilitate replacement of this module if we become aware of a better alternative.

After noise compensation, seven measurements were extracted from each data spectrum and stored. The first was amplitude, computed as the sum of the residual spectrum energy. This exploited Parseval's relation (Oppenheim and Schafer 1989, p. 574) to measure loudness after noise compensation. The remaining measurements described spectral characteristics. The frequency that bisected the energy in the power spectrum was saved as the median. The frequency corresponding to the largest energy value in the spectrum was saved as the mode.

Three estimates of "bandwidth" were saved. The minimum number of spectral bins needed to accumulate half of the total spectral energy was computed (including a fraction 
derived from linear interpolation); we designated this the concentration. The highest and lowest frequencies encountered in this integration were saved as the upper and lower frequencies; the difference between these provided a broader estimate of bandwidth, designated as spread. The ratio of total energy to the energy in the modal spectral bin was saved as the modewidth, the most compact bandwidth estimate of the three. We rescaled these three bandwidth estimators by dividing them by the sample interval represented by a single FFT block, so the resulting units were Hertz/s (otherwise, the same signal would have yielded different values when sampled at different rates or processed with different FFT sizes).

An analog of skewness, designated as asymmetry, was computed as (upper-median)/(upper-lower). Asymmetry varied between 0.0 (median equal to upper) and 1.0 (median equal to lower). Spectral asymmetry of 0.5 indicated a symmetrical density; so we later may shift these values by subtracting 0.5 from them to render the results more intuitive (this would not affect classifier performance).

The lists of short-term signal measurements were sorted to extract the upper quartile (75th percentile), median ( 50 th percentile), and lower quartile (25th percentile) values. When the computed index for one of the quartiles had a fractional component, the nearest values were used to linearly interpolate the desired value. The mode was estimated by finding the most tightly grouped set of five consecutive values, and selecting the middle of these. The quartiles were used to compute spread (upper quartile-lower quartile) and asymmetry (upper quartile-median)/(upper quartile-lower quartile). These statistics were analogous to the standard deviation and skewness, but they performed better. Amplitude was treated differently from the other short-term measurements. Its magnitude was arbitrary, so we divided mode and spread by the median to render them dimensionless. A total of 27 statistics resulted from these calculations:

- Amplitude: mode/median, spread/median, asymmetry

- Frequency Mode: mode, median, spread, asymmetry

- Frequency Median: mode, median, spread, asymmetry

- Spectral Spread: mode, median, spread, asymmetry

- Spectral Concentration: mode, median, spread, asymmetry

- Spectral Modewidth: mode, median, spread, asymmetry

- Spectral Asymmetry: mode, median, spread, asymmetry

Nonparametric correlations were computed among the short-term measurements, to quantify relationships among time, amplitude and frequency. We employed the Spearman Rank-Order Correlation (Press, W. H. et al. (1989), Numerical Recipes in C, Cambridge Univ. Press, pp. 507-509), and utilized the deviation of the sum-squared difference of ranks from its expected value, scaled in standard deviations. A large negative value indicated strong positive correlation, a large positive value indicated strong negative correlation (a sign change might be introduced later to ease interpretation). The 15 statistics resulting from these calculations were:

- Time-Amplitude Deviance

- Time-Frequency Mode Deviance

- Time-Frequency Median Deviance 
- Time-Spectral Spread Deviance

- Time-Spectral Concentration Deviance

- Time-Spectral Modewidth Deviance

- Time-Spectral Asymmetry Deviance

- Amplitude-Frequency Mode Deviance

- Amplitude-Frequency Median Deviance

- Amplitude-Spectral Spread Deviance

- Amplitude-Spectral Concentration Deviance

- Amplitude-Spectral Modewidth Deviance

- Amplitude-Spectral Asymmetry Deviance

- Frequency Median-Spectral Spread Deviance

- Frequency Median-Spectral Asymmetry Deviance

To measure "flat" frequency contours, which were often important in distinguishing among odontocete whistles, we timed the longest section in the signal exhibiting minimal change in frequency mode (maxflat). We computed the fraction of neighboring signal blocks in which the latter had more energy than the former (attack fraction), and in which the latter had a higher frequency median than the former (upsweep fraction). We also computed the average of all changes in frequency median (upsweep mean), and the average absolute value (sweep mean) of such changes.

Each short-term spectrum also contributed to two cumulative power spectra. One averaged all of the short-term spectra; this produced the marginal spectral density of the spectrographic representation of signal, the total spectrum. The second accumulated energy from the loudest element of each residual spectrum, the modal spectrum. Figure 4 exhibits the relationship of the total spectrum (frequency marginal energy density) and amplitude envelope (time marginal energy density) to a noise compensated signal. The dark regions represent the portions of these densities that concentrate $75 \%$ of the total signal energy. These cumulative spectra were summarized with the same spectral statistics as the short-term spectra. This produced 6 total spectrum and 6 modal spectrum statistics: medians, modes, spreads, concentrations, modewidths, and asymmetries. In total, 91 fields were produced by the feature extraction program for each sound.

\section{Classification Performance}

Two techniques were used to quantify the usefulness of these acoustic features for distinguishing among species. The first was a classical linear classifier (Morrison 1976, ch. 6), which would be optimal if the species differed in their group means, but shared a common multivariate normal dispersion (common covariance matrix). This was applied to a subset of the data consisting of isolated sound elements; it produced $73 \%$ correct classification (208 errors for 784 sounds). The distribution of mistakes is illustrated by the bubble graph in Figure 5. Most of the errors were located in a square at the lower left corner of the plot, which indicated confusion of one baleen whale sound for another. A weaker tendency was incorrect identification of some baleen whale sounds as seals.

Linear classification analysis of all sounds revealed poorer performance: only $50 \%$ correct (1037 errors for 2104 sounds). Figure 6 indicates the distribution of errors. Confusion 
among baleen whale sounds was again an important feature, but the horizontal banding in the plot indicated that a few species are responsible for most of the confusion. This structure is partly an artifact of sample sizes: more heavily sampled species will of course produce more incorrect classifications (these bubbles are not scaled to account for species sample size). This did not appear to be a complete explanation, and this phenomenon will be studied at greater length in the future.

An alternative technique for classifying these sounds utilized tree-based models (Clark and Pregibon 1992, ch. 9). This technique recursively partitioned the data, using a single variable at each binary split. At each point in the tree (called a node), a measure of diversity called "deviance" can be computed. It is defined as:

deviance $=-2 \sum_{k=1}^{N} y_{i k} \log \left(p_{i k}\right), y_{i k}=1$ if the $k^{\text {th }}$ individual is of class $i, 0$ otherwise; $p_{i k}=$ the probability that the $k^{\text {th }}$ individual is of class $i$, estimated as the fraction of individuals in the node of class $i$.

This is equivalent to minus twice the log-likelihood function. Each interior node (including initial node containing all sounds) is split such that the residual deviance of the resulting pair of nodes is maximally reduced. Thus, the process of splitting results in successively "purer" nodes, with the process terminating when a node is sufficiently pure or there are insufficient individuals in the node to support another split. This process provides both a simple technique for classifying unknown sounds (a series of true/false questions) and clues to the important variables for diagnosis. It also accommodates diversity within a class: if a species produces two or more distinct types of sounds, a tree-based analysis will not be compromised (unlike a linear or quadratic classifier).

Figure 7 exhibits the tree-based classifier for the isolated sounds. The vertical distance associated with each split graphically depicts the reduction in deviance achieved by that split. The initial split was based on the median short-term spectral concentration; the next two splits were based on the median frequency of the total spectrum and the spectral concentration of the frequency modulation spectrum. This analysis permits a species' sounds to be split into more than one "leaf," depending upon their relationships to the other sounds in the sample. Correct classification was $85 \%$; this is nearly a $50 \%$ reduction in misclassification relative to the linear classifier. A tree-based classification analysis of all sounds yielded $66 \%$ correct classification (figure 8). Tyack, Fristrup and McIntosh (submitted) have shown that similar analyses of signature whistles in young bottlenose dolphins correctly identified the individual for $90 \%$ of the sounds tested.

The linear classifier had one advantage over the tree-based classifier: it provided a measure of similarity to help judge the correctness of the identification. The tree-based technique must be augmented to provide this capability, using some distance metric generated from the terminal groupings. A straightforward adaptation would be to calculate a sample covariance matrix for each terminal grouping, and use Mahalanobis distance to measure the similarity of an unknown to that group. This adaptation, and tests of aiternative classification schemes (quadratic classifier, $\mathrm{kNN}$ voting, hybrid designs), will be pursued further. 


\section{Literature Cited}

Caldwell, M. C., D. K. Caldwell and P. L. Tyack 1990. The Bottlenose Dolphin: recent progress in research. Academic Press, San Diego.

Clark, L. A., and D. Pregibon 1992. Statistical Models in S. J. M. Chambers and T. J. Hastie (eds.). Wadworth and Brooks/Cole, Pacific Grove, CA.

Morrison, D. F. 1976. Multivariate Statistical Analysis, $2^{\text {nd }}$ ed.. McGraw-Hill Inc., New York.

Tyack, P. L., K. Fristrup and J. S. McIntosh submitted. Signature whistle development in three captive bottlenose dolphins Tursiops truncatus. Animal Behavior.

Watkins, William A., Kurt Fristrup, and Mary Ann Daher 1991. Marine animal SOUND database. Technical Report WHOI-91-21, Woods Hole Oceanographic Institution, Woods Hole, MA 02543, 51 pp.

Watkins, William A, and Douglas Wartzok 1985. Sensory biophysics of marine mammals. Marine Mammal Science 1:219-260. 
Figure 1. Noise compensation performance: higher levels of "signal excess" represent better performance. Each vertical line represents the range of performance achieved with a fixed multiplier order statistic and varying magnification. The leftmost vertical line starts at its minimum with a magnifier of 8 , and terminates at the intersection with the diagonal line with a multiplier of 160 . The initial and terminal values of subsequent settings of multiplier order statistic are indicated by the intersections with diagonal lines on the left and right. The range of magnification decreases with increasing order statistic, but the maximum performance is clearly bracketed in each case. 


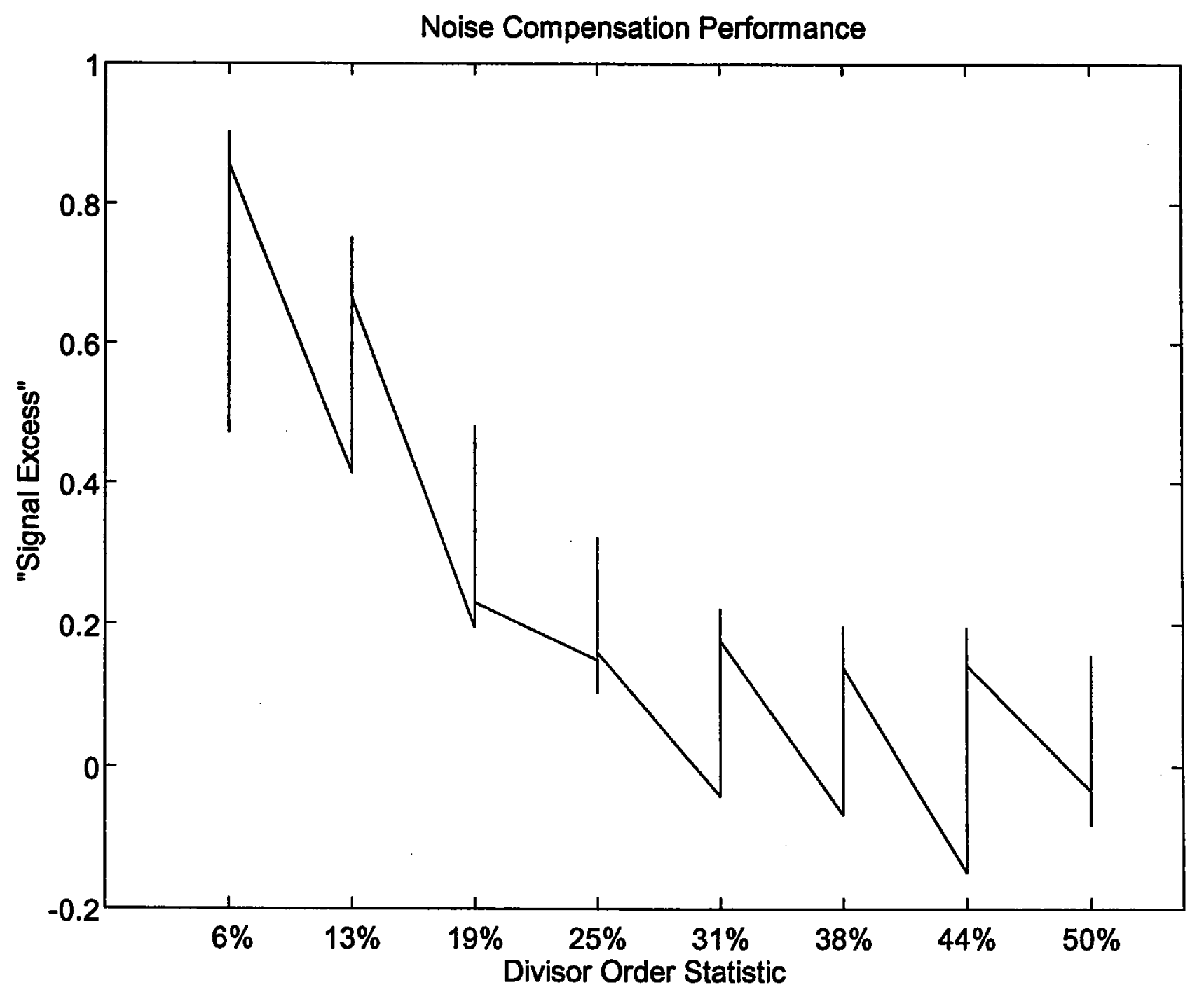


Figure 2. Noise compensation performance: this sequence of spectrographs illustrates a recording of Lagenodelphis hosei, the Fraser's dolphin. The first panel is the unmodified signal. The second illustrates the same signal processed using the older, fixed compensation algorithm. The third the illustrates the effect of processing with the newer, adaptive compensation algorithm. The pronounced speckling in all spectrographs represents echolocation clicks by the dolphins. 
Raw Spectrograph: Lagenodelphis Whistles

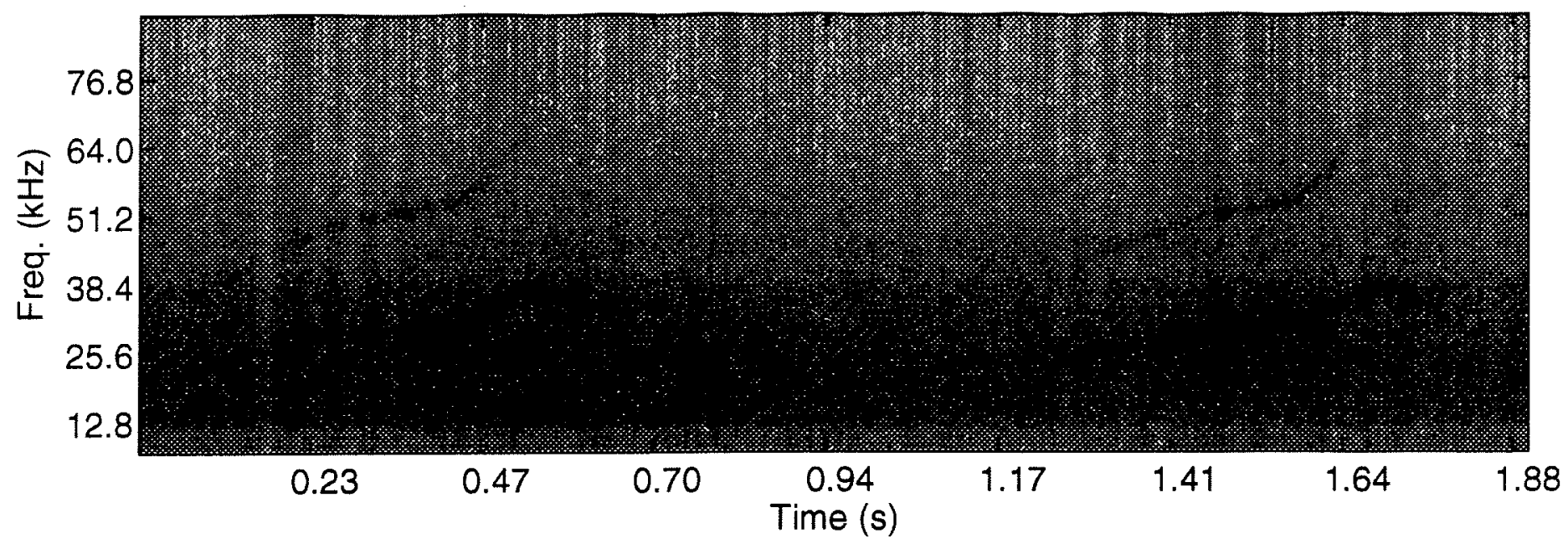

Fixed Compensation

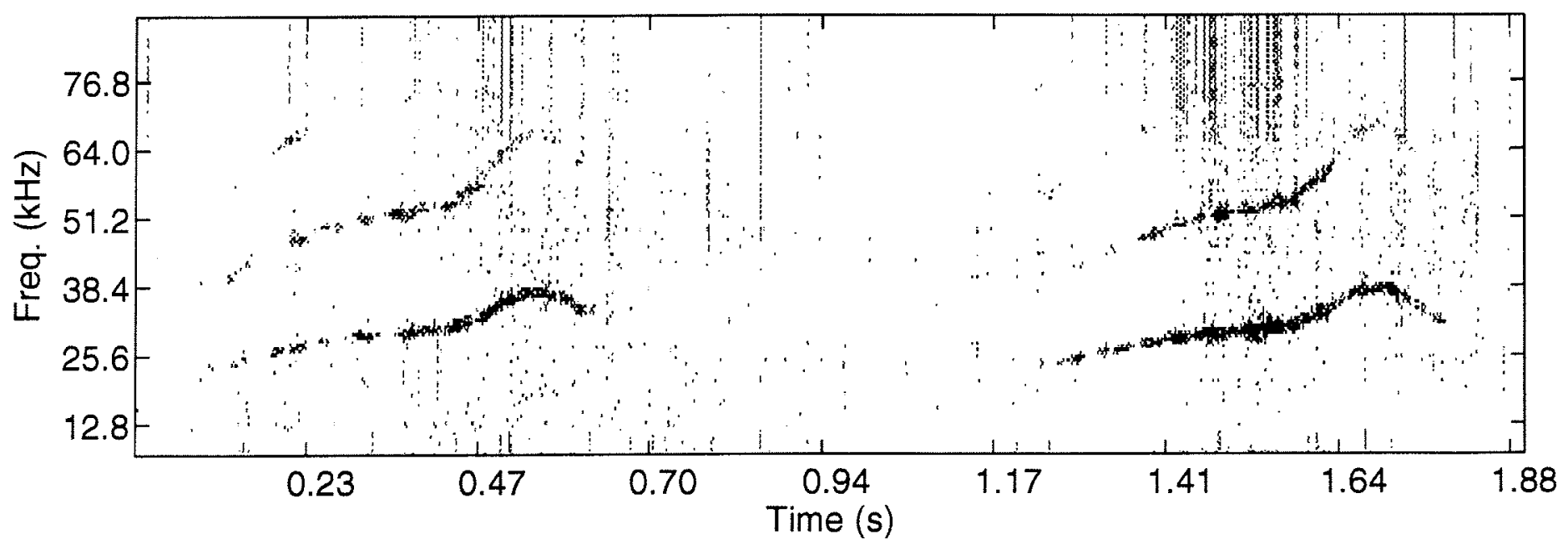

Adaptive Compensation

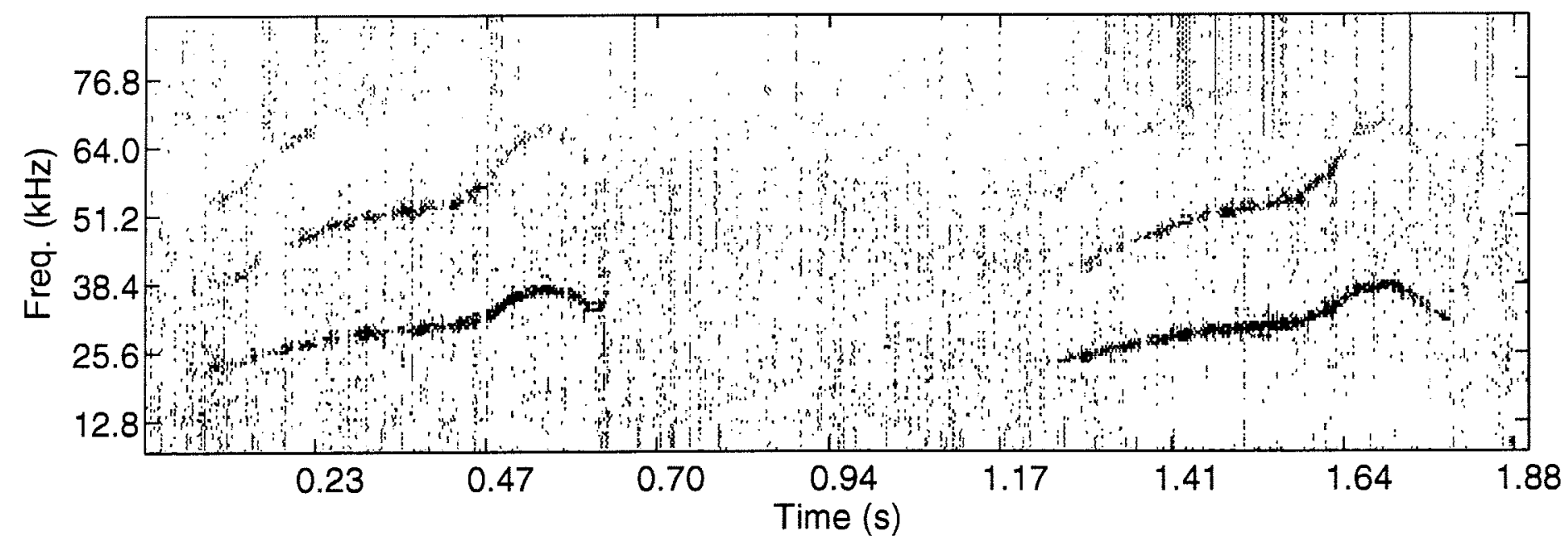


Figure 3. Noise compensation performance: this sequence of spectrographs illustrates a recording of Eubalaena glacialis, the northern right whale. The first panel is the unmodified signal. The second illustrates the same signal processed using the older, fixed compensation algorithm. The third the illustrates the effect of processing with the newer, adaptive compensation algorithm. Note the preservation of a faint, introductory moan in the third panel, near the left edge. 
Raw Spectrograph: Eubalaena moans

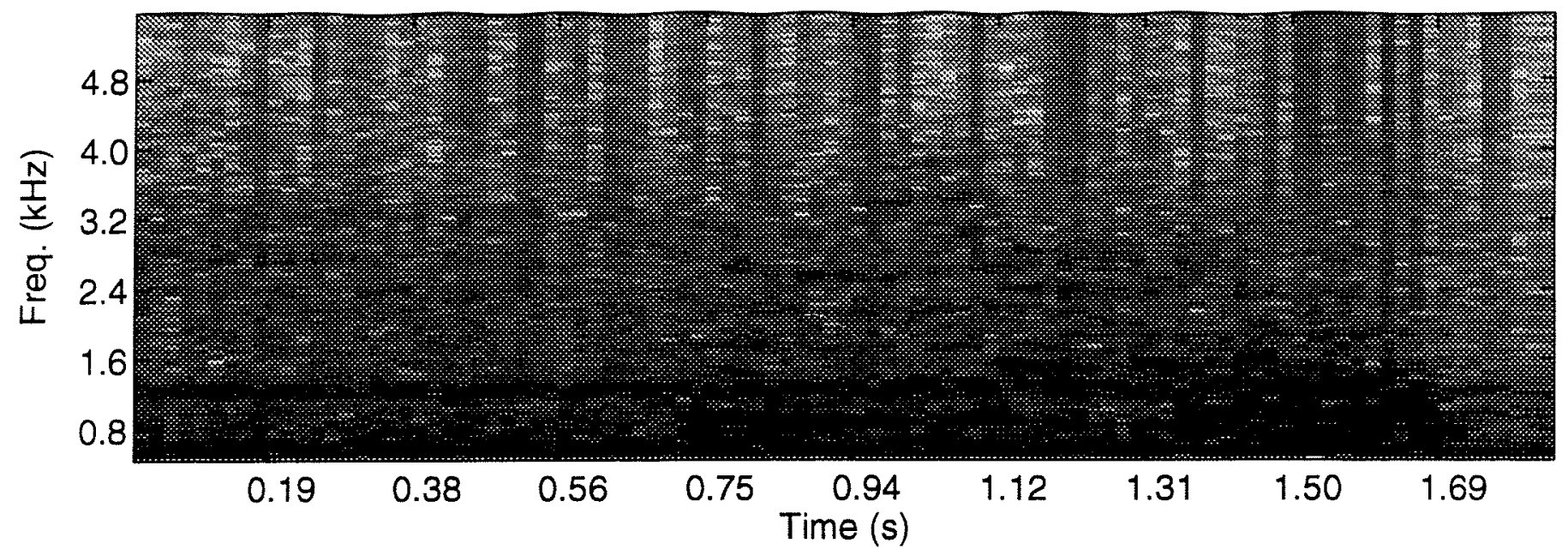

Fixed Compensation

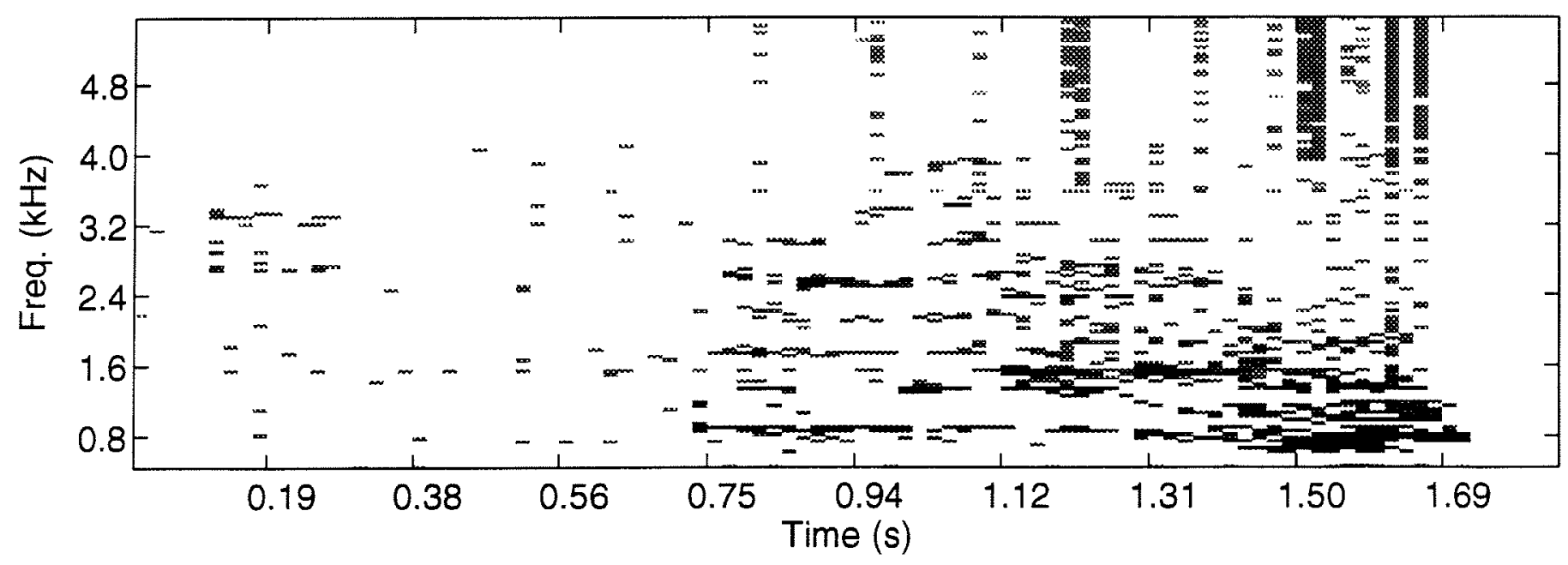

Adaptive Compensation

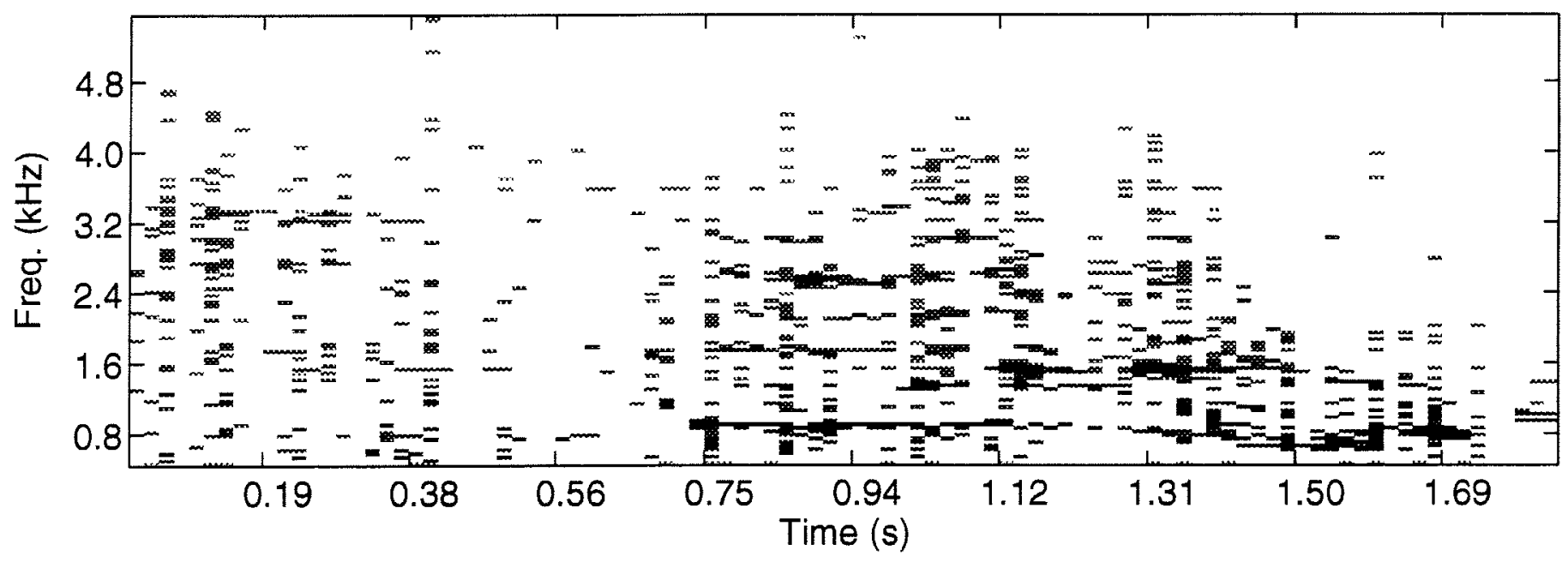


Figure 4. The time and frequency marginal energy densities, in relation to the spectrograph that produced them. The dark areas of the energy densities indicate the portions included in the calculation of concentration, upper, and lower values. The original spectrograph was of very poor quality, not useable for classifier training. Note the leakage of low frequency noise energy, and the appearance of this energy in the shaded portions of the marginal distributions. 

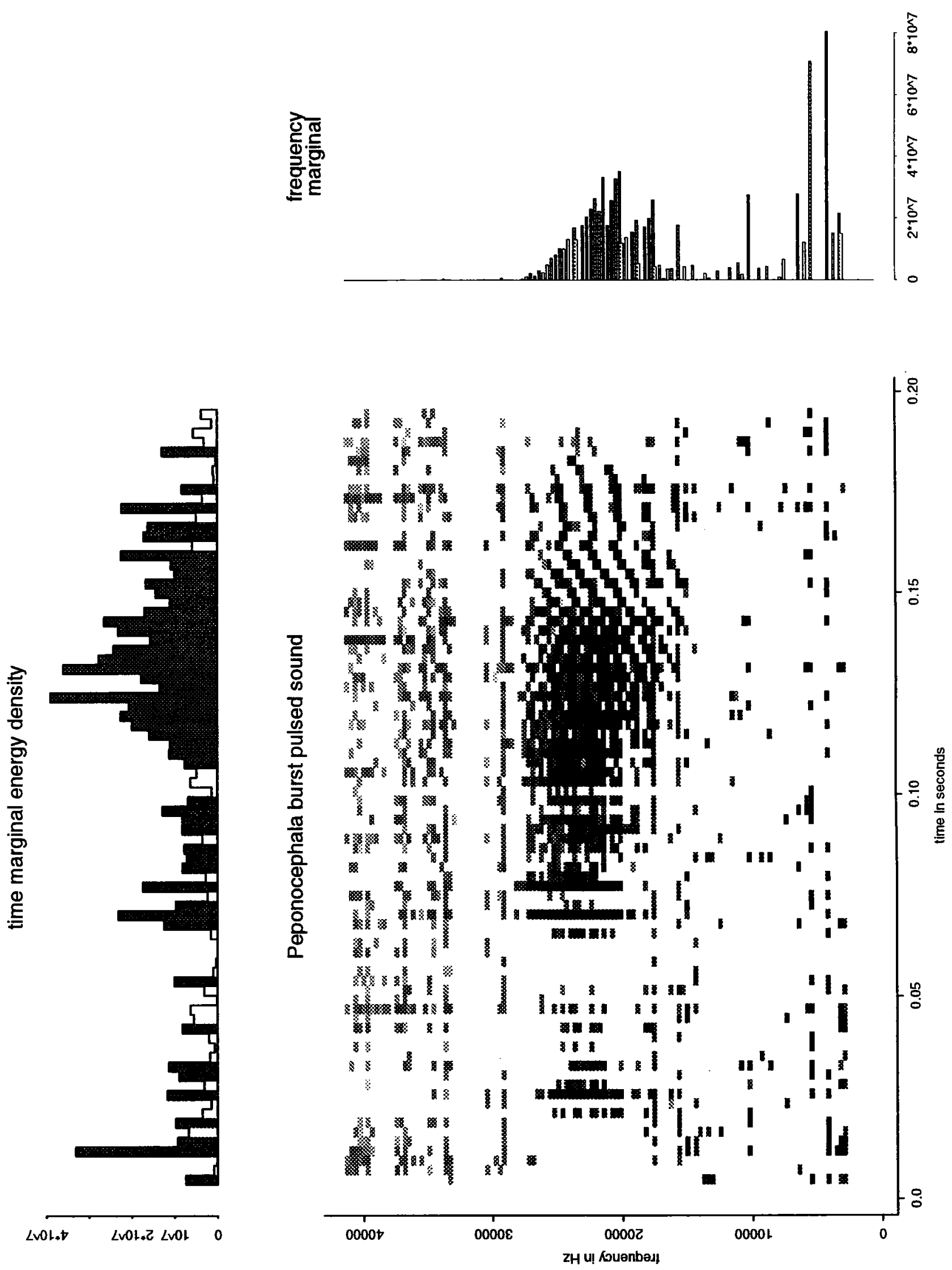
Figure 5. Linear classifier performance, isolated sounds: the $\mathrm{x}$ and $\mathrm{y}$ axes represent the species tested, the area of the circle represents the number of mistakes. The numeric ordering closely follows systematic ordering (historical relatedness). Numbers 1-8 are baleen whale species; numbers 9-22 are toothed whale species, 23-31 are seals, and number 32 is a manatee. Specifically,

1. Balaena mysticetus

2. Eubalaena glacialis

3. Eubalaena australis

4. Eschrichtius robustus

5. Balaenoptera acutorostrata

6. Balaenoptera borealis

7. Balaenoptera physalus

8. Megaptera novaeangliae

9. Physeter catodon

10. Delphinapterus leucas

11. Monodon monoceros

12. Peponocephala electra

13. Steno bredanensis

14. Delphinus bairdii

15. Delphinus delphis

16. Grampus griseus

17. Lagenorhynchus acutus

18. Globicephala macrorhynchus

19. Globicephala melaena

20. Orcinus orca

21. Pseudorca crassidens

22. Phocoena phocoena

23. Arctocephalus forsteri

24. Eumetopias jubatus

25. Odobenus rosmarus

26. Phoca fasciata

27. Phoca largha

28. Ommatophoca rossi

29. Erignathus barbatus

30. Halichoerus grypus

31. Leptonychotes weddellii

32. Trichechus manatus 
Linear classifier errors (208/784): isolated sounds area of circles is proportional to number of errors

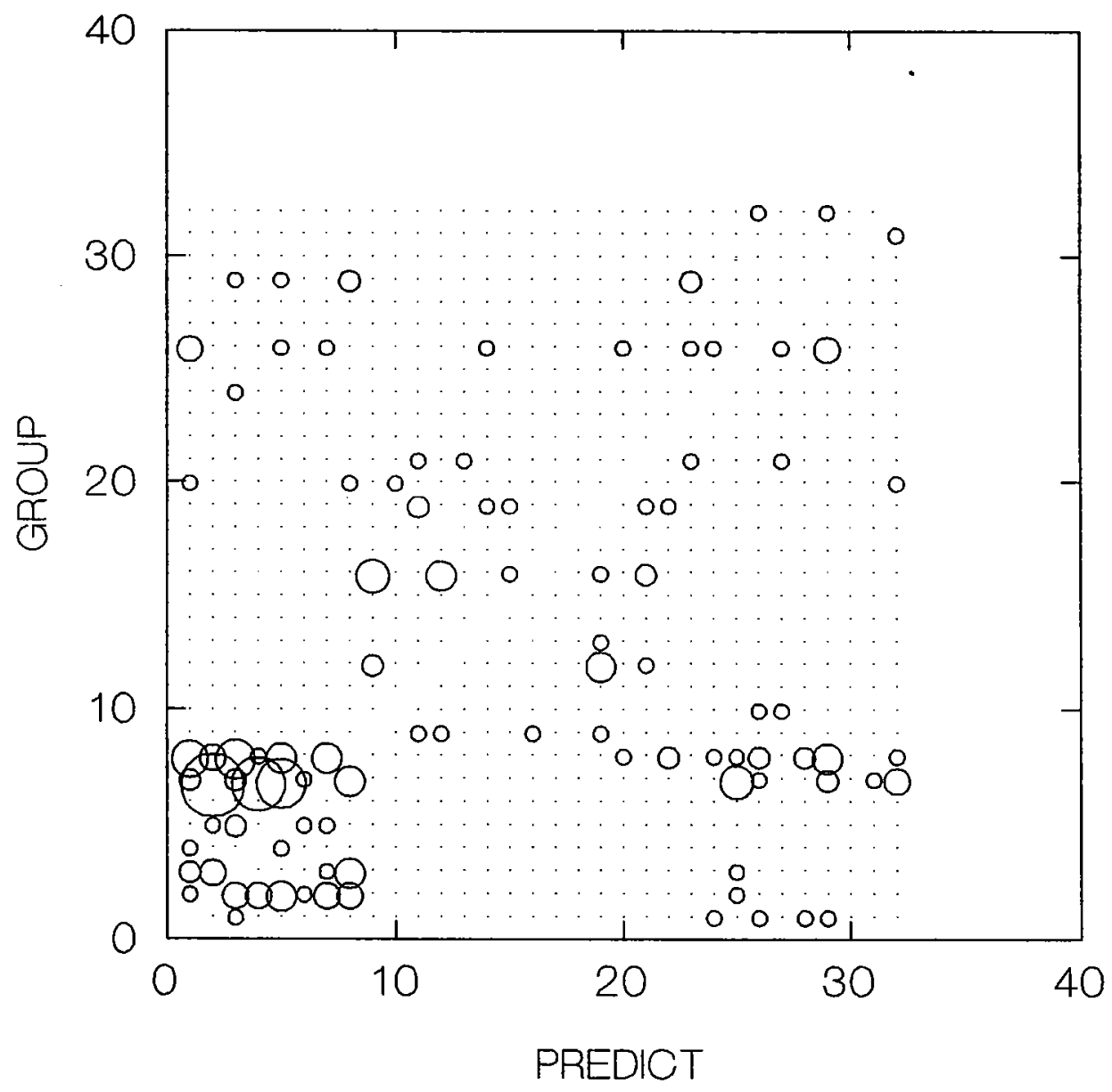


Figure 6. Linear classifier performance, all sounds: the $\mathrm{x}$ and $\mathrm{y}$ axes represent the species tested, the area of the circle represents the number of mistakes. The numeric ordering closely follows systematic ordering (historical relatedness). Numbers 1-9 are baleen whale species; numbers 9-39 are toothed whale species, 40-53 are seals, and number 54 is a manatee. Specifically,

1. Balaena mysticetus

2. Caperea marginata

3. Eubalaena glacialis

4. Eubalaena australis

5. Eschrichtius robustus

6. Balaenoptera acutorostrata

7. Balaenoptera borealis

8. Balaenoptera physalus

9. Megaptera novaeangliae

10. Physeter catodon

11. Delphinapterus Leucas

12. Monodon monoceros

13. Peponocephala electra

14. Sotalia

15. Sousa

16. Stenella attenuata

17. Stenella clymene

18. Stenella coeruleoalba

19. Stenella longirostris

20. Steno bredanensis

21. Tursiops catalania

22. Tursiops truncatus

23. Cephalorhynchus commersonii

24. Cephalorhynchus heavisidii

25. Delphinus bairdii

26. Delphinus delphis

27. Grampus griseus

28. Lagenodelphis hosei

29. Lagenorhynchus acutus

30. Lagenorhynchus albirostris

31. Globicephala sp.

32. Globicephala macrorhynchus

33. Globicephala melaena

34. Globicephala scammoni

35. Orcinus orca

36. Pseudorca crassidens

37. Phocoena phocoena

38. Neophocaena phocaenoides

39. Inia geoffrensis

40. Arctocephalus forsteri

41. Eumetopias jubatus

42. Odobenus rosmarus

43. Phoca fasciata

44. Phoca groenlandica

45. Phoca hispida

46. Phoca largha

47. Ommatophoca rossi

48. Cystophora cristata

49. Erignathus barbatus

50. Halichoerus grypus

51. Leptonychotes weddellij

52. Enhydra lutris

53. Trichechus manatus 
Linear classifier errors (1037/2104): all sounds area of circles is proportional to number of errors

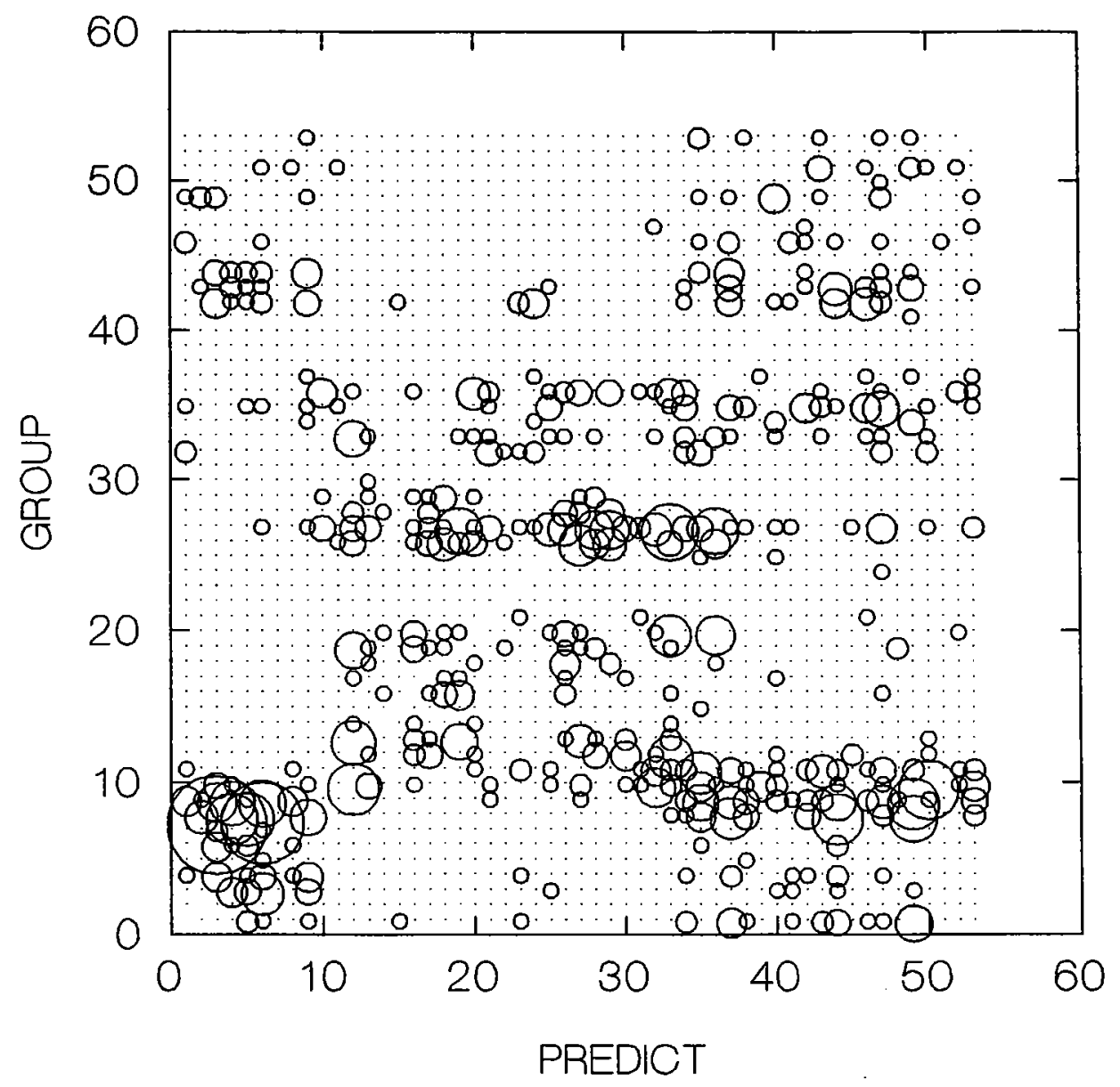


Figure 7. Tree-based classification, isolated sounds: the minuscule labels at each interior node describe the criterion that was used to split the sounds at that node into two subnodes. The terminal nodes ("leaves") of the tree, located at the bottom of the figure, are labeled with a code describing the identities of the dominant fractions of the sounds in those nodes. The code reflects the systematic hierarchy. The translations are:

CONCmed:median st concentration

TSmed:total spectrum median frequency

MSmed:modal spectrum median frequency

ERGmxmd:maximum/median amplitude

FMEDSPRDr:median freq. X spread corr.

EGDmodw: amplitude modewidth

MODWmod:mode of st modewidth

FMSmod:FM spectrum mode

TSmod:total spectrum mode

MSmodw:modal spectrum mode

ATAKfrac:attack fraction

FMSmed: FM spectrum median

SPRDsprd: spread of st spread

AMSmod:AM spectrum mode

UPSWfrac:upsweep fraction

TSmodw:total spectrum modewidth

ASYMmod:modal st asymmetry

AMSupp:AM spectrum upper frequency

MODWmed:median st modewidth

TSasym:total spectrum asymmetry

FMSconc:FM spectrum concentration

SPRDasym:asymmetry of st spread

AASYMr:amplitude $X$ st asymm. corr.

FMSsprd: FM spectrum spread

AFMODWr:amplitude $\mathrm{X}$ st modewidth corr.

TSupp:total spectrum upper frequency

MSupp:modal spectrum upper frequency

st $==$ short term
AA1A: Balaena mysticetus

AA3A: Eubalaena glacialis

AA3B: Eubalaena australis

AB1A: Eschrichtius robustus

AC1A: Balaenoptera acutorostrata

AC1B: Balaenoptera borealis

AC1F: Balaenoptera physalus

AC2A: Megaptera novaeangliae

BA2A: Physeter catodon

BB1A: Delphinapterus leucas

BB2A: Monodon monoceros

BD10A: Peponocephala electra

BD17A: Steno bredanensis

BD3A: Delphinus bairdii

BD3B: Delphinus delphis

BD4A: Grampus griseus

BD6A:. Lagenorhynchus acutus

BE3B: Globicephala macrorhynchus

BE3C: Globicephala melaena

BE7A: Orcinus orca

BE9A: Pseudorca crassidens

BF2A: Phocoena phocoena

CA1F: Arctocephalus forsteri

CA3B: Eumetopias jubatus

CB1A: Odobenus rosmarus

CC12F: Phoca fasciata

CC12L: Phoca largha

CC14A: Ommatophoca rossi

CC2A: Erignathus barbatus

CC3A: Halichoerus grypus

CC5A: Leptonychotes weddellii

DB1B: Trichechus manatus 


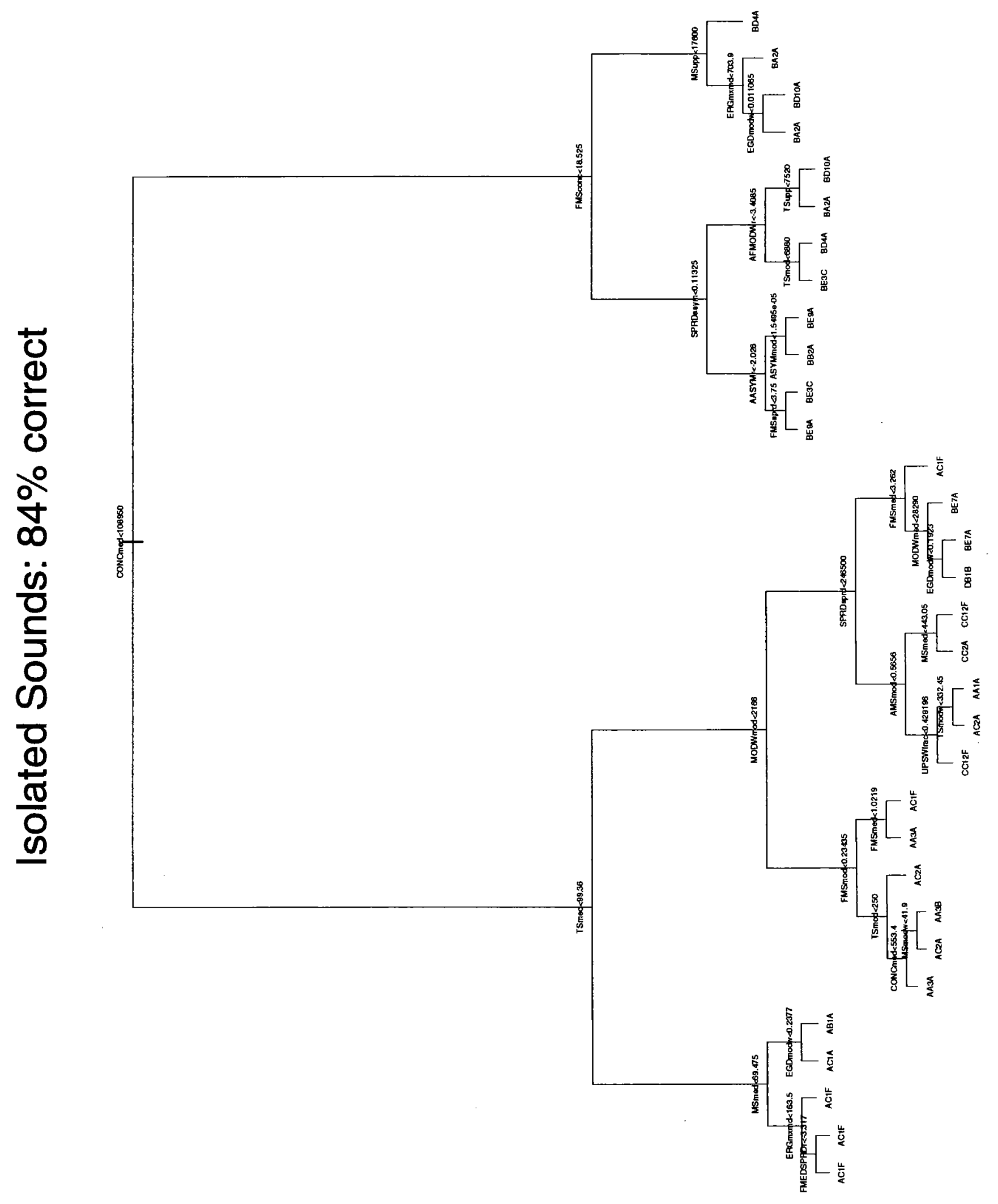


Figure 8. Tree-based classification, all sounds: the minuscule labels at each interior node describe the criterion that was used to split the sounds at that node into two subnodes. The terminal nodes ("leaves") of the tree, located at the bottom of the figure, are labeled with a code describing the identity of the dominant fractions of the sounds in those nodes. The code reflects the systematic hierarchy. The translations are:

MODWmed:median st modewidth

MSmod:modal spectrum mode

ERGmxmd:maximum/median amplitude

SPRDsprd:spread of st spread

FMSmod: FM spectrum mode

MSmed:modal spectrum median

AMSmod:AM spectrum mode freq. MODWsprd:spread of st modewidth MSmodw:modal spectrum modewidth SWPabs:mean absol. delta freq. CONCasym:asymm. of st concent. FMEDmed:median st median freq. Maxflat:see text description SPRDmed:median st spread ATAKfrac:attack fraction FMSsprd: FM spectrum spread SPRDmod:mode of st spread TSsprd:total spectrum spread EGDconc:amplitude concentration FMSconc: FM spectrum concent. FMEDASYMr:median freq. X asymmetry corr. TSupp:total spectrum upp. freq. FMSupp: FM spectrum upper freq. EGDsprd:amplitude spread

AFMODWr:amplitude $\mathrm{X}$ modewidth correlation FMEDSPRDr:med.freq. X spread correlation TSmed:total spect. median freq. MODWmod:mode of st modewidth ASYMasym:asymmetry of st asymm. FMODmed:median of st mode freq. CONCmod:mode of st concent. FMEDmod:mode of st median freq. AMSconc:concent. of st asymm. ASYMmed:median st asymmetry UPSWfrac:upsweep fraction ERGCV:amplitude coeff. of var. st $==$ short term
AA1A: Balaena mysticetus AA2A: Caperea marginata AA3A: Eubalaena glacialis AA3B: Eubalaena australis AB1A: Eschrichtius robustus AClA: Balaenoptera acutorostrata AC1B: Balaenoptera borealis AC1F: Balaenoptera physalus AC2A: Megaptera novaeangliae BA2A: Physeter catodon BB1A: Delphinapterus leucas BB2A: Monodon monoceros BD10A: Peponocephala electra BD12: Sotalia BD13: Sousa BD1SA: Stenella attenuata BD15B: Stenella clymene BD15C: Stenella coeruleoalba BD15L: Stenella longirostris BD17A: Steno bredanensis BD19B: Tursiops catalania BD19D: Tursiops truncatus BD1A: Cephalorhynchus commersonii BDIC: Cephalorhynchus heavisidii BD3A: Delphinus bairdii BD3B: Delphinus delphis BD4A: Grampus griseus BD5A: Lagenodelphis hosei BD6A: Lagenorhynchus acutus BD6B: Lagenorhynchus albirostris BE3: Globicephala sp. BE3B: Globicephala macrorhynchus BD3C: Globicephala melaena BE3D: Globicephala scammoni BE7A: Orcinus orca BE9A: Pseudorca crassidens BF2A: Phocoena phocoena BF6A Neophocaena phocaenoides BG2A: Inia geoffrensis CA1F: Arctocephalus forsteri CA3B: Eumetopias jubatus CB1A: Odobenus rosmarus CC12F: Phoca fasciata CC12G: Phoca groenlandica CC12H: Phoca hispida CC12L: Phoca largha CC14A: Ommatophoca rossi CC1A: Cystophora cristata CC2A: Erignathus barbatus CC3A: Halichoerus grypus CC5A: Leptonychotes weddellii CD1A: Enhydra lutris

DB1B: Trichechus manatus 


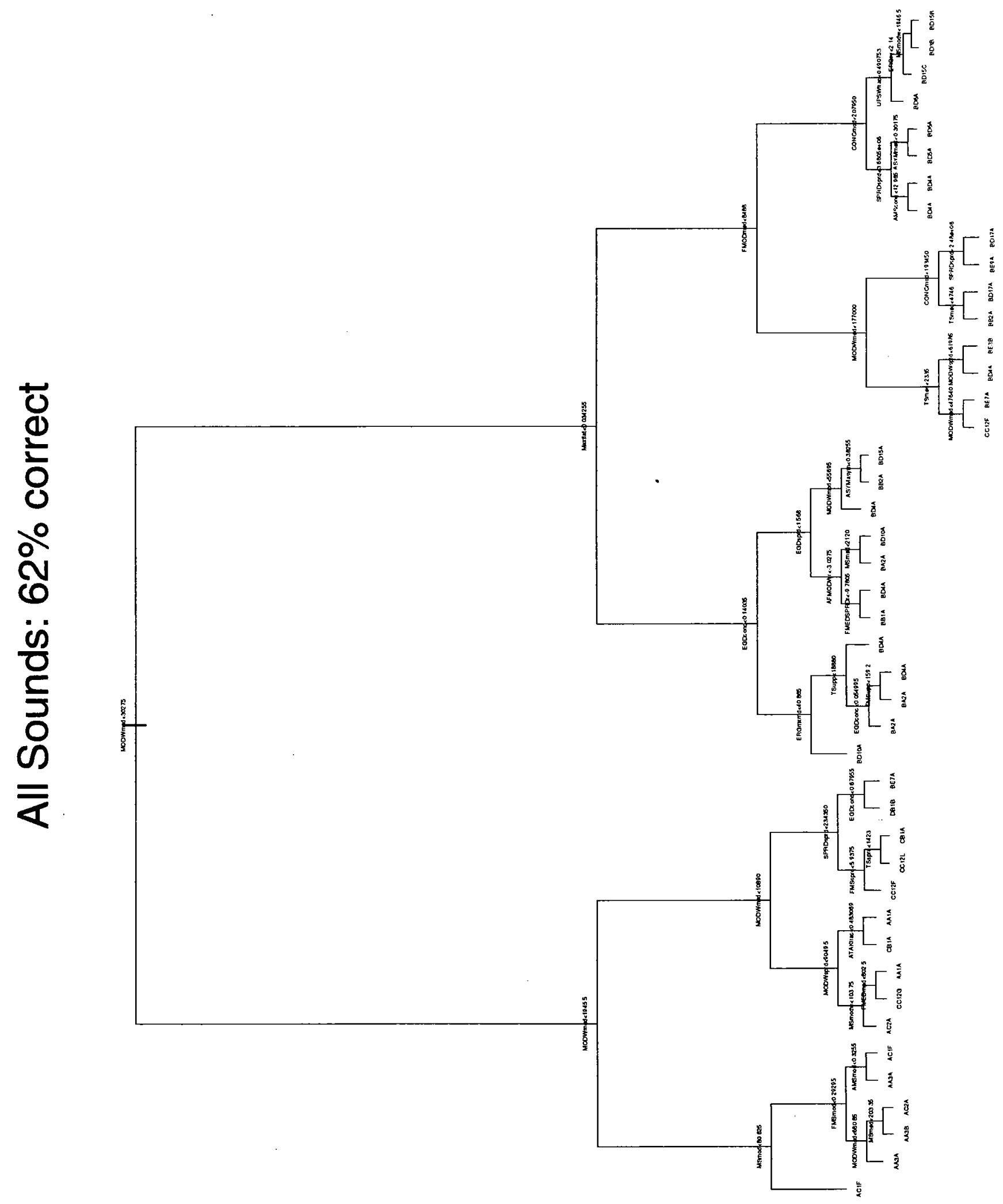




\section{DOCUMENT LIBRARY}

Distribution List for Technical Report Exchange - May 5, 1994

University of California, San Diego

SIO Library 0175C (TRC)

9500 Gilman Drive

La Jolla, CA 92093-0175

Hancock Library of Biology \& Oceanography

Alan Hancock Laboratory

University of Southern California

University Park

Los Angeles, CA 90089-0371

Gifts \& Exchanges

Library

Bedford Institute of Oceanography

P.O. Box 1006

Dartmouth, NS, B2Y 4A2, CANADA

Commander

International Ice Patrol

1082 Shennecossett Road

Groton, CT 06340-6095

NOAA/EDIS Miami Library Center 4301 Rickenbacker Causeway

Miami, FL 33149

Library

Skidaway Institute of Oceanography

10 Ocean Science Circle

Savannah, GA 31411

Institute of Geophysics

University of Hawaii

Library Room 252

2525 Correa Road

Honolulu, HI 96822

Marine Resources Information Center

Building E38-320

MIT

Cambridge, MA 02139

Library

Lamont-Doherty Geological Observatory

Columbia University

Palisades, NY 10964

Library

Serials Department

Oregon State University

Corvallis, OR 97331

Pell Marine Science Library

University of Rhode Island

Narragansett Bay Campus

Narragansett, RI 02882
Working Collection

Texas A\&M University

Dept. of Oceanography

College Station, TX 77843

Fisheries-Oceanography Library

151 Oceanography Teaching Bldg.

University of Washington

Seattle, WA 98195

Library

R.S.M.A.S.

University of Miami

4600 Rickenbacker Causeway

Miami, FL 33149

Maury Oceanographic Library

Naval Oceanographic Office

Building 1003 South

1002 Balch Blvd.

Stennis Space Center, MS 39522-5001

Library

Institute of Ocean Sciences

P.O. Box 6000

Sidney, B.C. V8L 4B2

CANADA

Library

Institute of Oceanographic Sciences

Deacon Laboratory

Wormley, Godalming

Surrey GU8 5UB

UNITED KINGDOM

The Librarian

CSIRO Marine Laboratories

G.P.O. Box 1538

Hobart, Tasmania

AUSTRALIA 7001

Library

Proudman Oceanographic Laboratory

Bidston Observatory

Birkenhead

Merseyside L43 7 RA

UNITED KINGDOM

IFREMER

Centre de Brest

Service Documentation - Publications BP 7029280 PLOUZANE

FRANCE 


\begin{tabular}{|c|c|c|c|}
\hline $\begin{array}{l}\text { REPORT DOCUMENTATION } \\
\text { PAGE }\end{array}$ & 1. REPORT NO. WHOI-94-13 & 2 & 3. Recipient's Accession No. \\
\hline \multirow{2}{*}{\multicolumn{3}{|c|}{$\begin{array}{l}\text { 4. Title and Subtitle } \\
\text { Marine Animal Sound Classification }\end{array}$}} & $\begin{array}{l}\text { 5. Report Date } \\
\text { October } 1993\end{array}$ \\
\hline & & & $\overline{6}$ \\
\hline \multicolumn{3}{|c|}{ 7. Author(s) $\quad$ Kurt M. Fristrup and William A. Watkins } & $\begin{array}{l}\text { 8. Performing Organization Rept. No. } \\
\text { WHOI-94-13 }\end{array}$ \\
\hline \multicolumn{3}{|c|}{ 9. Performing Organization Name and Address } & 10. Project/Task/Work Unit No. \\
\hline \multicolumn{3}{|c|}{$\begin{array}{l}\text { Woods Hole Oceanographic Institution } \\
\text { Woods Hole, Massachusetts } 02543\end{array}$} & $\begin{array}{l}\text { 11. Contract(C) or Grant(G) No. } \\
\text { (C) N-00140-90-D-1979 } \\
\text { (G) }\end{array}$ \\
\hline \multirow{2}{*}{\multicolumn{3}{|c|}{$\begin{array}{l}\text { 12. Sponsoring Organization Name and Address } \\
\text { Office of Naval Research through the Naval Undersea Warfare Center Division }\end{array}$}} & $\begin{array}{l}\text { 13. Type of Report \& Period Covered } \\
\text { Technical Report }\end{array}$ \\
\hline & & & 14. \\
\hline
\end{tabular}

15. Supplementary Notes

This report should be cited as: Woods Hole Oceanog. Inst. Tech. Rept., WHOI-94-13.

16. Abstract (Limit: 200 words)

Software was developed to measure characteristics of marine animal sounds (AcouStat). These measurements proved effective for classifying sounds in several contexts: identifying species, quantifying the repertoire of a single species, and identifying individuals. The sound measures included statistics for aggregate bandwidth, intensity, duration, amplitude modulation, frequency modulation, center frequency, and interactions among these variables. Classification analysis based on these measures suggests they adequately characterize the variability of bioacoustic signals for many problems. Correct classification to species was as high as $85 \%$, and correct classification of dolphin whistles to individual was $90 \%$.

17. Document Analysis a. Descriptors marine

sound

classification

b. Identifiers/Open-Ended Terms

c. COSATI Field/Group

18. Availability Statement

Approved for public release; distribution unlimited.

\begin{tabular}{|l|l|}
\hline $\begin{array}{c}\text { 19. Security Class (This Report) } \\
\text { UNCLASSIFIED }\end{array}$ & $\begin{array}{c}21 . \text { No. of Pages } \\
33\end{array}$ \\
\hline 20. Security Class (This Page) & 22. Price \\
\hline & $\begin{array}{l}\text { OPTIONAL FORM 272 (4-77) } \\
\text { (Formerly NTIS-35) } \\
\text { Department of Commerce }\end{array}$ \\
\hline
\end{tabular}

